\title{
Characterization by FTIR and Ampicillin Release in Collagen-Polyurethane Hydrogels Coupled with Metal-Organic Frameworks
}

\author{
Nadia J. Amaya-Chantaca ${ }^{1}$, Irene D. Reyes-Ruiz ${ }^{1}$, Denis A. Cabrera-Munguía ${ }^{1}$, Tirso E. Flores-Guía ${ }^{1}$, Lucía F. \\ Cano-Salazar ${ }^{1}$, Laura Espindola-Serna ${ }^{2} \&$ Jesús A. Claudio Rizo ${ }^{1} *$
}

${ }^{1}$ Universidad Autónoma de Coahuila, Facultad de Ciencias Químicas, Ing J.Cardenas Valdez S/N, República, 25280 Saltillo, Coahuila, México.
${ }^{2}$ Universidad Autónoma de la Ciudad de México, Calle Dr. García Diego 168, Doctores, Cuauhtémoc, 06720, Ciudad de México, México.
E-mail: jclaudio@ uadec.edu.mx ${ }^{I *}$

DOI: 10.38177/ajast.2020.4214

\section{ABSTRACT}

\begin{abstract}
In this work, the capacity of encapsulation and subsequent release of the drug ampicillin was evaluated, for this, two different hydrogels were made by the microemulsion method, having different crosslinking agent based on polyurethane: $\mathrm{P}(\mathrm{IPDI})$ and $\mathrm{P}(\mathrm{HDI})$, the metal-organic framework (MOF) that was used inside the two composite hydrogels was MIL-53, which is a derivative of the hydrothermal synthesis of terephthalic acid and Al (III) ions. Characterization tests were carried out on the hydrogels, the FTIR sprectra were obtained to evaluate the encapsulation of the drug and the quantification by UV-Vis spectrophotometry to monitor the mass of ampicillin released in the two systems studied. Hydrogels based on C-P(HDI)-MIL53 show higher intermolecular interactions with encapsulated ampicillin, observed by the variation in the intensities of the FTIR signals related to the - $\mathrm{NH},-\mathrm{OH}$, amide I and II bonds that make up the systems; in addition this type of hydrogel also exhibits a higher release of ampicillin than C-P(IPDI)-MIL53 hydrogel. These hydrogels can be studied as 3D culture systems or materials for wound healing, thus avoiding the formation of bacterial infections.
\end{abstract}

Keywords: Hydrogels, Chemical crosslinking, Collagen, Polyurethane, MOF, Ampicillin.

\section{Introduction}

Hydrogels are three-dimensional networks swollen in water comprised of polymers that have the ability to absorb it, thanks to the polarity of their functional groups, some of the polar groups of these compounds are: $-\mathrm{CONH}_{2},-\mathrm{OH},-\mathrm{COOH}, \mathrm{SO}_{3} \mathrm{H}$, among others. Hydrogels are a versatile platform for drug delivery due to their ability to encapsulate and protect drugs, providing a both controlled and sustained release of the encapsulated drugs, depending on the properties of the hydrogel ${ }^{[1-5]}$. In general, the mechanical properties of the hydrogel must be regulated to support the load of the encapsulated drug, thus preventing the swollen 3D matrix from breaking; it is also important to regulate the swelling and degradation behaviors of the matrix so that the drug has a release favorable profile. In this sense, research focused on the development of hydrogels that meet these requirements is a recent boom area.

Hydrogels derived from biopolymers such as collagen show excellent therapeutic performance due to their high biocompatibility and because the degradation products of these matrices are not toxic to the host organism. However, it is necessary to regulate the mechanical properties and the swelling/degradation behaviors for these matrices to be used as drug delivery systems. In this sense, crosslinking of collagen with polyurethane represents an alternative to regulate these properties ${ }^{[6-8]}$.

The reactive isocyanate groups of the polyurethane react with the primary amino groups of collagen to generate crosslinking urea bonds; the generation of these bonds allows to regulate the mechanical properties and the swelling/degradation profiles of the hydrogels obtained; besides allowing the encapsulation of therapeutic molecules such as dexamethasone, observing controlled release profiles of such drug ${ }^{[6-8]}$. Recently, the incorporation of inorganic particles with the capacity to adsorb bioactive molecules within the 
hydrogels based on biopolymers, represents a strategy to ensure a controlled release of the therapeutic, improving the biomedical performance of the hydrogel ${ }^{[9-13]}$. In this sense, the metal-organic frameworks (MOFs), which are coordination polymers based on metal ions and organic ligands, attract attention for this application due to their high surface area and adsorptive capacity. MOFs have continued to be used for encapsulation and controlled release of various molecules of therapeutic interest ${ }^{[14-18]}$. With this in mind, the generation of composite hydrogels that include MOF with encapsulated drugs represents a potential alternative for the design of materials with improved biomedical application.

Ampicillin is a broad-spectrum beta-lactam antibiotic; the development of hydrogels that have the ability to release this antibiotic would represent an alternative to generate hydrogels with potential application in the healing of chronic wounds. With this in mind, in the present work it is contemplated to adsorb this antibiotic in the MOF MIL-53, (derived from the hydrothermal synthesis of Al (III) ions with terephatyl acid); and the subsequent generation of composite hydrogels (polyurethane crosslinked collagen matrices with encapsulated MOF/ampicillin). The study evaluates the effect of varying the chemical structure of the polyurethane crosslinker, (based on polyethylene glycol $\left(1000 \mathrm{~g} \mathrm{~mol}^{-1}\right)$ with hexamethylenediisocyanate $(\mathrm{P}$ (HDI)) or isophorone diisocyanate P (IPDI)), on the encapsulation capacity of MOF/ampicillin and the release of the antibiotic. The encapsulation capacity of MOF/ampicillin inside the collagen-polyurethane was evaluated by FTIR and the release of ampicillin was monitored by UV-vis spectrophotometry.

\section{Experimental Methodology}

\subsection{Synthesis of hydrogels based on collagen-polyurethane coupled with MOF/ampicillin}

Type I collagen from bovine tendon was extracted as previously reported ${ }^{[19]}$. Crosslinkers based on aqueous polyurethane dispersions were synthesized using polyethylene glycol (PEG) (1000 g/mol), hexamethylenediisiocinate $(\mathrm{P}(\mathrm{HDI}))$ or isophoronadiisocyanate (P(IPDI)), as reported in the literature ${ }^{[7]}$. For the synthesis of MIL53, aluminum nitrate nonahydrate $\left(\mathrm{Al}\left(\mathrm{NO}_{3}\right)_{3} \cdot 9 \mathrm{H}_{2} \mathrm{O}\right)$ are mixed with terephthalic acid (HOOC- $\mathrm{C}_{6} \mathrm{H}_{4}-\mathrm{COOH}, \mathrm{BDC}$ ) in a $1: 2$ molar ratio, respectively, in $50 \mathrm{~mL}$ of water distilled until complete dissolution. The above mixture is placed in a $100 \mathrm{~mL}$ capacity hydrothermal synthesis reactor, which is placed in an oven at $200^{\circ} \mathrm{C}$ for 3 days. The reactor is then allowed to cool to room temperature, the white precipitate is filtered under vacuum and washed with distilled water to neutral $\mathrm{pH}$. The solid is dried at $80^{\circ} \mathrm{C}$ for $24 \mathrm{~h}$. Ampicillin adsorption on MIl-53 was carried out by adding $500 \mu \mathrm{g}$ of antibiotic in a $50 \%$ ethanol solution and over $1 \mathrm{mg}$ of MOF; the mixture was left to stand for $1 \mathrm{~h}$ and was subsequen tly filtered to recover MOF/ampicillin.

The composite hydrogels were synthesized using the plate microemulsion method, for this: $1 \mathrm{ml}$ of a standardized collagen solution $(6 \mathrm{mg} / \mathrm{mL}$ ) was crosslinked using a 15 weight $\%$ (wt.\%) ratio of crosslinking agent $\mathrm{P}$ (HDI) or $\mathrm{P}$ (IPDI), as the case may be. The crosslinking reaction was carried out at $\mathrm{pH} 7.0$ at $4{ }^{\circ} \mathrm{C}$ for $2 \mathrm{~h}$. Subsequently, once the viscosity of the systems increased, $1 \mathrm{mg}$ of MIL-53 containing $500 \mu \mathrm{g}$ de ampicillin adsorbed was added and vortexed until correct dispersion. The formation of the composite 
hydrogels loaded with ampicillin was carried out at $37^{\circ} \mathrm{C}$ for $18 \mathrm{~h}$. In Table 1 , the compositions and designations of the composite hydrogels prepared in this work are presented.

Table 1. Formulation of the composite hydrogels under study

\begin{tabular}{|c|c|c|c|c|}
\hline $\begin{array}{c}\text { Bovine Type I } \\
\text { collagen (6 } \\
\mathbf{m g} / \mathbf{m L}) \mathbf{m L}\end{array}$ & $\begin{array}{c}\text { Type of } \\
\text { crosslinker used in } \\
\mathbf{1 5} \mathbf{w t . \%} \text { ratio }\end{array}$ & $\begin{array}{c}\text { MOF } \\
\text { MIL-53/ } \\
\mathbf{m g}\end{array}$ & Ampicillin/ $\mathbf{\mu g}$ & Hydrogel designation \\
\hline $\mathbf{1}$ & $\mathrm{P}(\mathrm{HDI})$ & 1 & ------ & C-P(HDI)-MIL53 \\
\hline $\mathbf{1}$ & $\mathrm{P}(\mathrm{IPDI})$ & 1 & ------- & C-P(IPDI)-MIL53 \\
\hline $\mathbf{1}$ & $\mathrm{P}(\mathrm{HDI})$ & 1 & 500 & C-P(HDI)-MIL53/A \\
\hline $\mathbf{1}$ & $\mathrm{P}(\mathrm{IPDI})$ & 1 & 500 & C-P(IPDI)-MIL53/A \\
\hline
\end{tabular}

\subsection{Characterization of composite hydrogels}

To assay the chemical structure of composite hydrogels and the MOF/ampicillin encapsulation, ATR -FTIR spectra with a Frontier, Perkin Elmer system, were recorded on fresh hydrogel composites at $16 \mathrm{~cm}^{-1}$ of resolution in a range from 3600 to $650 \mathrm{~cm}^{-1}$, using an average of 16 scans.

\subsection{Evaluation of ampicillin release}

For drug release, a composite hydrogel of each formulation was taken and placed in a $50 \mathrm{ml}$ Falcon tube, adding $10 \mathrm{~mL}$ of $1 \mathrm{X}$ phosphate buffer solution (PBS), and then were taken $3 \mathrm{~mL}$ aliquots in a time of 2 and 5 hours, respectively, at $37^{\circ} \mathrm{C}$. Ampicillin quantification was performed using a calibration curve of known concentration standards. Detection readings were made at $410 \mathrm{~nm}$ employing a Lambda 35 Perkin-Elmer (UV-VIS) spectrophotometer.

\subsection{Data analysis}

The mean and standard deviation (SD) are presented for each data set. Data sets were compared using analysis of variance (ANOVA). The difference of the means was checked with a Fisher Minimum Significant Difference test and was considered statistically significant at level $p<0.05$.

\section{Results and Discussion}

The study of the encapsulation capacity of molecules with therapeutic interest in collagen-polyurethane hydrogels, determines the interaction of the chemical structure of the encapsulated component with the chemical structures of the polymeric networks that make up the hydrogel. In this sense, for the drug ampicillin $(500 \mu \mathrm{g} /$ hydrogel $)$ in hydrogels based on collagen-polyurethane, it was evaluated by FTIR. The FTIR spectra of the studied systems are shown in figure 1: 

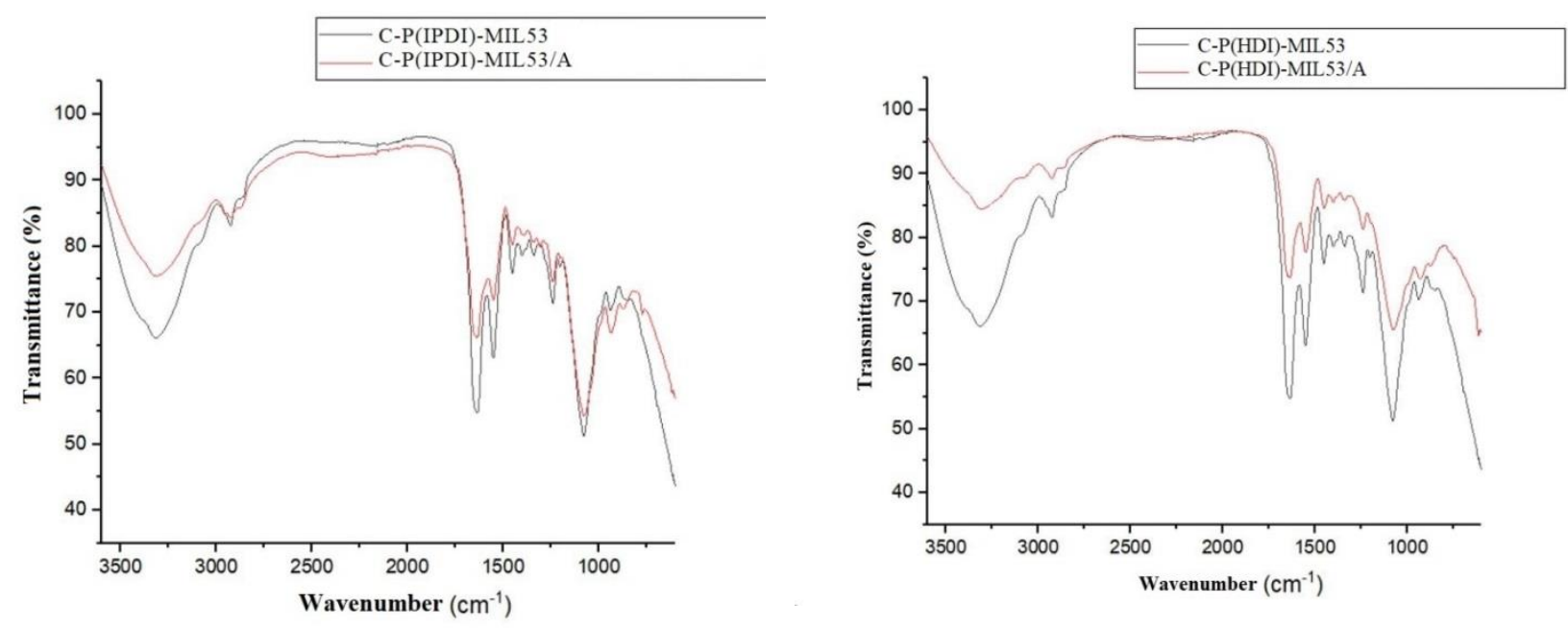

Figure 1. Evaluation of the encapsulation capacity of MOF MIL-53 with adsorbed ampicillin on collagen-polyurethane hydrogels by FTIR

The chemical structure of the starting hydrogel was considered as a study variable in order to know its influence on the encapsulation capacity and subsequent release of the drug; for this, two different hydrogels were made by modifying the crosslinker based on polyurethane: P (IPDI) rigid polymeric structure with aliphatic rings present in its skeleton, and P(HDI) aliphatic linear flexible polymeric structure; the MOF that was used for the two systems was MIL-53 derived from the hydrothermal synthesis of terephthalic acid and $\mathrm{Al}$ (III) ions.

The encapsulation and/or loading of amoxicillin in composite matrices can be elucidated by observing the intensities of the bands among $3600-3000 \mathrm{~cm}^{-1}$ corresponding to the $-\mathrm{NH}$ and $\mathrm{OH}$ bonds of the polymeric components used such as collagen as base matrix and the crosslinking polyurethane; systems that include amoxicillin show a decrease in this band indicating the presence of short-range intermolecular interactions such as hydrogen bridges between these matrix polymer bonds and the $\mathrm{N}-\mathrm{H}, \mathrm{NH}-\mathrm{C}=\mathrm{O}$ and $\mathrm{OH}$ groups present in the chemical structure of the antibiotic.

On the other hand, also a decrement in the intensities of the vibrations associated with the amide I and II modes of collagen at 1640 and $1550 \mathrm{~cm}^{-1}$, respectively, is appreciated when the drug is encapsulated in the matrix (C-P(HDI)-MIL53/A and C-P(IPDI)-MIL53/A spectra), again indicating the presence of short-range interaction with the amide functional group of collagen. The band around $920 \mathrm{~cm}^{-1}$ in all the studied hydrogels is associated to the Al-O bond present in the incorporated MIL-53 structure; where it is important to note that in C-P(HDI)-MIL53 a decrease in the intensity of this signal is observed with respect to the C-P(IPDI)-MIL53; indicating that the aliphatic linear polymeric matrix experiences higher adsorption interactions with the MOF MIL-53; related to the freedom of movement that exists in the polymeric system; this is not so evident in the P(IPDI)-MIL53 hydrogel since the stiffness of the aliphatic rings in the crosslinker polymer skeleton limits the adsorption interactions with MOF. Finally, systems that include amoxicillin C-P(HDI)-MIL53/ and C-P(IPDI)-MIL53/A show a small band at $855 \mathrm{~cm}^{-1}$ related to Al-N 
interactions, showing that the metal-organic structure has the ability to adsorb the coupled drug and also to interact with the $-\mathrm{NH}$ groups of the polymers that make up matrix.

The effect of these intermolecular interactions related to encapsulation in the matrix in the hydrogel state is decisive for the release capacity of the encapsulated drug ${ }^{[15-18]}$. The crosslinked matrix with $\mathrm{P}(\mathrm{HDI})$ shows greater effect to regulate the encapsulation of ampicillin in a uniform way, because there are no aliphatic regions related to steric impediments that tend to agglomerate ampicillin, as in the case of the material that includes P(IPDI). Also, it is interesting to highlight that a high presence of these intermolecular interactions with the encapsulated components could be associated with the drug release capacity by modifying the $\mathrm{pH}$, temperature or ionic strength, factors associated with the alteration of said intermolecular interactions stimulating the release of the drug ${ }^{[9,16-18]}$.

In this way, the work contemplated the monitoring of the mass of ampicillin released in the systems under study by UV-Vis spectrophotometry, for which an ampicillin calibration curve was performed. The calibration equation was used to evaluate the amount of ampicillin released in systems incubated at $37^{\circ} \mathrm{C}$ for a time of 2 and 5 hours. The calibration equation determined for the study is:

Absorbance $_{(410 \mathrm{~nm})}=\left(5 \times 10^{-5}\right) \mu \mathrm{g}$ Ampicillin +0.0015 , with a linear correlation factor of 0.9204 .

Figure 2 reveals the percentage of ampicillin released (considering an initial mass of $500 \mu \mathrm{g}$ ), the results indicate that $63 \pm 6 \%$ and $81 \pm 8 \%$ of ampicillin released for C-P(IPDI)-MIL53/A and C-P(HDI)-MIL53/A, respectively, at 2 hours; and $72 \pm 5 \%$ and $86 \pm 6 \%$ for C-P(IPDI)-MIL53/A and C-P(HDI)-MIL53/A, respectively at 5 hours of incubation. Statistically significant differences were found when comparing the ampicillin release values of C-P(HDI)-MIL53/A with respect to C-P(IPDI)-MIL53/A, at both incubation times evaluated.

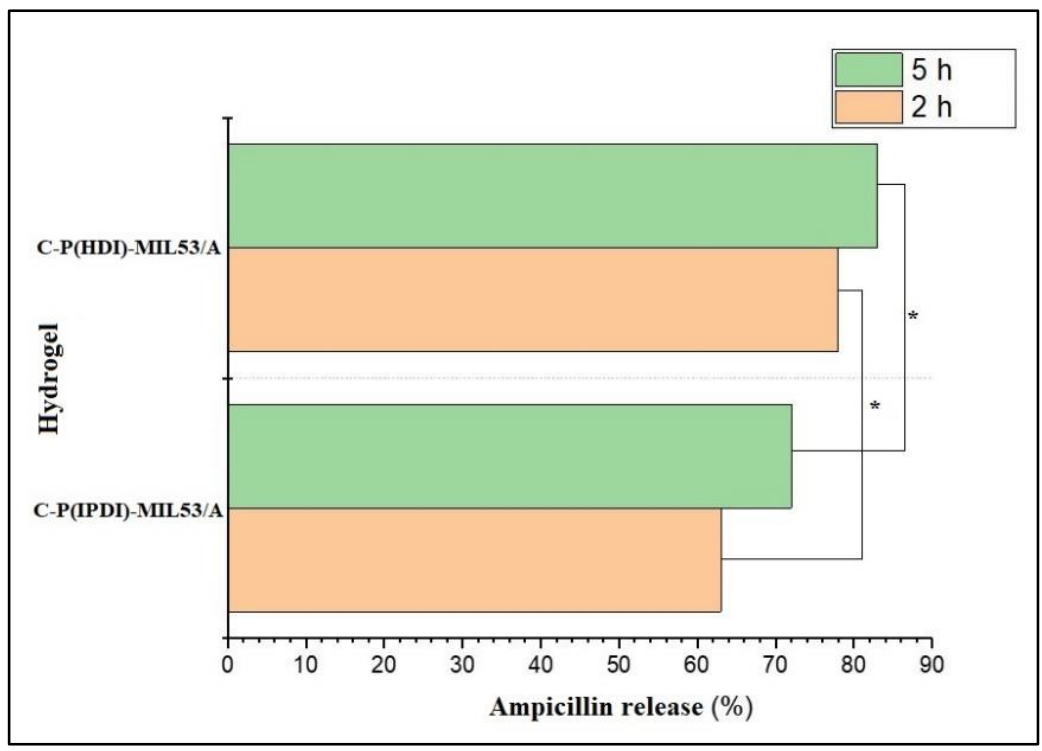

Figure 2. Results of ampicillin release from collagen-polyurethane and MIL-53 metal-organic frameworks based hydrogels 
The results indicate that the C-P(HDI)-MIL53 matrix shows increased release capacity of the encapsulated ampicillin. The release of ampicillin within these matrices is carried out by a swelling-diffusion process ${ }^{[9]}$, where the matrix in a first stage is swollen and the water adsorbed by the hydrogels alters the encapsulation balances of the drug, to later release it to the outside by a secondary process of diffusion. Therefore, matrices that have a high swelling capacity can promote rapid diffusion of encapsulated bioactive molecules. In the research group it has been reported that collagen matrices crosslinked by $\mathrm{P}(\mathrm{HDI})$-based polyurethanes tend to have higher swelling capacity than those derived from P(IPDI), thus promoting swelling and diffusion of the encapsulated drug. The water molecules that enter the matrix tend to destabilize the intermolecular interactions that keep the drug encapsulated, thus stimulating the release by swelling.

The chemical structure of the polyurethane crosslinker is decisive in the release capacity of the ampicillin coupled in the MOF MIL-53; the rigid cyclic regions present in the C-P(IPDI)-MIL53 prevents both the water molecules from entering the matrix; limiting the release capacity, however these regions also have a direct interaction with the chemical structure of the antibiotic that keeps it more strongly encapsulated providing a less release than in the C-P(HDI)-MIL53 hydrogel that does not have aliphatic cyclic regions. From a chemical point of view, it is interesting to control the release of the antibiotic due to the composition of the chemical structure of the hydrogel. This strategy can be exploited for biomedical applications where systems that retain the antibiotic are required to avoid contamination of systems with encapsulated cells, or for a sustained release that prevents the formation of infections when the hydrogel is used as a wound dressing for tissue healing for specific time.

\section{Conclusion}

The development of hydrogels that have the encapsulation capacity of drugs with therapeutic interest and subsequent release capacity of these, is a promising research topic to develop hydrogels with improved biomedical application. In this work, it studied the encapsulation and release capacity of ampicillin in hydrogels based on collagen-polyurethane and MIL-53. The chemical structure of polyurethane was modified to evaluate its effect on the study variables. Hydrogels based on C-P(HDI)-MIL53 show higher intermolecular interactions with encapsulated ampicillin, observed by the variation in the intensities of the FTIR signals related to the - $\mathrm{NH}$, -OH, amide I and II bonds that make up the systems; in addition this type of hydrogel also exhibits a higher release of ampicillin. The rigid aliphatic structures present in the C-P (IPDI) -MIL53 hydrogel limit the release of the drug, however they ensure the drug favorable encapsulation. These hydrogels can be studied as 3D culture systems or materials for wound healing, thus avoiding the formation of bacterial infections.

\section{References}

[1] G. S. Alvarez, C. Helary, A. M. Mebert, X. Wang, T. Coradin and M. F. Desimone, J. Mater. Chem. B, 2014, 2 , 4660 .

[2] D. Wallace, Adv. Drug Delivery Rev., 2003, 55, 1631-1649.

[3] M. R. Blatchley and S. Gerecht, Biomed. Mater., 2015, 10, 034001. 
[4] C. Caramella, B. Conti, T. Modena, F. Ferrari, M. C. Bonferoni, I. Genta, S. Rossi, M. L. Torre, G. Sandri, M. Sorrenti, L. Catenacci, R. Dorati and G. Tripodo, J. Drug Delivery Sci. Technol., 2016, 32, $206-228$.

[5] S. Hinderer, S. L. Layland and K. Schenke-Layland, Adv. Drug Delivery Rev., 2016, 97, $260-269$.

[6] T. D. Sargeant, A. P. Desai, A. S. Banerjee, A. Agawu and J. B. Stopek, Acta Biomater., 2012, 8, 124-132.

[7] J. A. Claudio-Rizo, M. Rangel-Argote, L. E. Castellano, J. Delgado, J. L. Mata-Mata, B. Mendoza-Novelo, Mater Sci Eng C, 2017, 79, 793-801.

[8] J. A. Claudio-Rizo, B. Mendoza-Novelo, J. Delgado, L. E. Castellano, J. L. Mata-Mata J L, Biomed Mater (Bristol), 2016, 11(3), 035016.

[9] M. Rangel-Argote, Jesús A. Claudio-Rizo, L. E. Castellano, A. Vega-González, J. L. Mata-Mata, B. Mendoza-Novelo, RSC Adv, 2017, 7, 10443.

[10] M. Antman-Passig, O. Shefi, Nano Letters, 2016, 16(4), 2567-2573.

[11] M. F. Desimone, C. Helary, S. Quignard, I. B. Rietveld, I. Bataille, G. J. Copello, G. Mosser, M. M. Giraud-Guille, J. Livage, A. Meddahi-Pelle, T. Coradin, ACS Appl Mater Interfaces, 2011, 3(10), 3831-3838.

[12] A. K. Miri, N. Muja, N. O. Kamranpour, W. C. Lepry, A. R. Boccaccini, S. A. Clarke, S. N. Nazhat, Biomaterials, 2016, 85: 128-41.

[13] Z. Xia, M. M. Villa, M. Wei, J Mater Chem B, 2014, 2(14), 1998-2007.

[14] W. H. Chen, W. C. Liao, Y. S. Sohn, M. Fadeev, A. Cecconello, R. Nechushtai R, Adv Funct Mater 2018, 28(8), 1-9.

[15] J. Xiao, S. Chen, J. Yi, H. F. Zhang, G. A. Ameer, Adv Funct Mater, 2017, 27(1), 1-10.

[16] Y. Yu, G. Chen, J. Guo, Y. Liu, J. Ren, T. Kong, Mater Horiz, 2018, 5(6), 1137-42.

[17] A. Mazloom-Jalali, Z. Shariatinia, I. A. Tamai, S. R. Pakzad, J. Malakootikhah, Int J Biol Macromol, 2020, $153,421-32$.

[18] D. Han, Y. Li, X. Liu, B. Li, Y. Han, Y Zheng, Chem Eng J, 2020, 125194. 